\title{
STARLIKE, CONVEX, CLOSE-TO-CONVEX, SPIRALLIKE, AND $\Phi$-LIKE MAPS IN A COMMUTATIVE BANACH ALGEBRA WITH IDENTITY
}

BY

\author{
L. F. HEATH AND T. J. SUFFRIDGE ${ }^{1}$
}

\begin{abstract}
Let $C(X)$ be the space of continuous functions on a compact $T_{2}$-space $X$ where each point of $X$ is a $G_{\delta}$. If $F: B \rightarrow C(X)$ is a biholomorphic (in the sense that $F$ and $F^{-1}$ are Frechet differentiable) map of $B=\{f \mid\|f\|<1\}$ onto a convex domain with $D F(0)=I$, then $F$ is Lorch analytic (i.e., $D F(f)(g)=a_{f} g$ for some $a_{f} \in C(X)$ ).

Let $R$ be a commutative Banach algebra with identity such that the Gelfand homomorphism of $R$ into $C(\mathscr{T})$ is an isometry. Starlike, convex, close-to-convex, spirallike and $\Phi$-like functions are defined in $B=\{x \in$ $R \mid\|x\|<1\}$ for $L$-analytic functions in $B$ and they are related to associated complex-valued holomorphic functions in $\Delta=\{z \in C|| z \mid<1\}$.
\end{abstract}

Introduction. In $\$ \S 2-7$, let $R$ be a commutative Banach algebra over the complex numbers with identity (denoted by 1 ) and let $\Re$ be the space of maximal ideals in $R$. Then $\Re$ is a compact, $T_{2}$-space where the topology is the weakest topology on $\Re$ such that the Gelfand transformation $x(M)$ of $x$ is a continuous function on $\mathfrak{T}$. Assume further that the Gelfand homomorphism of $R$ into $C(\mathscr{T})$ is an isometry; i.e., $\|x\|=\sup \{|x(M)| \mid M$ $\in \mathfrak{N}\}$ for all $x \in R$. Let $B=\{x \in R \mid\|x\|<1\}$ and $\Delta=\{z \in \mathbf{C}|| z \mid<1\}$.

If $D$ is an open set in $R$, we say $F: D \rightarrow R$ is $L$-analytic in $D$ if for each $x \in D$, there is $F^{\prime}(x) \in R$ such that

$$
\lim _{h \rightarrow 0} \frac{\left\|F(x+h)-F(x)-h F^{\prime}(x)\right\|}{\|h\|}=0
$$

[11]. Thus it is clear that $L$-analytic functions are Fréchet differentiable. If $F$ : $B \rightarrow R$ is $L$-analytic in $B$, then for each $x \in B, F(x)=\sum_{n=0}^{\infty} a_{n} x^{n}$ where $a_{n} \in R$ and the series converges uniformly on $\|x\|<\rho<1$ [7, Theorems 3.19.1 and 26.4.1]. If $F: D \rightarrow R$ is $L$-analytic in $D$ and for each $y \in F(B)$, there is an open neighborhood $V$ of $y$ such that $F^{-1}$ exists and is $L$-analytic

\footnotetext{
Presented to the Society, January 4, 1978 under the title Convex Fréchet differentiable map of the unit ball of $C(X)$ into $C(X)$; received by the editors June 8, 1977.

AMS (MOS) subject classifications (1970). Primary 46J10, 30A32.

Key words and phrases. Starlike, convex, close-to-convex, spirallike, $\Phi$-like, $F$-holomorphic, $L$-analytic.

${ }^{1}$ The research of the second author was supported in part by the National Science Foundation under grant number MCS 75-06971 A01.
} 
in $V$, then we say that $F$ is locally bianalytic in $B$. If $F$ is univalent (one-to-one) and locally bianalytic in $B$, we say that $F$ is bianalytic in $B$. If $F$ is $L$-analytic in $B$, then for each $M \in \mathscr{N}$, there is an associated holomorphic function $F_{M}: \Delta \rightarrow \mathrm{C}$ defined by $F_{M}(z) \equiv F(z 1)(M)$ for all $z \in \Delta$. If $F(x)=$ $\sum_{n=1}^{\infty} a_{n} x^{n}$ is $L$-analytic in $B$, then we write $F(x) / x$ for the $L$-analytic function $\sum_{n=1}^{\infty} a_{n} x^{n-1}$.

\section{Preliminary lemmas.}

Lemma 2.1. Let $V: B \times I \rightarrow B$ be L-analytic in $B$ for each $t \in I=[0,1]$, $V(0, t)=0$ for all $t \in I, V(x, 0)=x$ for all $x \in B$. If $\lim _{t \rightarrow 0^{+}}(x-$ $V(x, t)) /(x t)=U(x)$ exists and is L-analytic in $B$, then $\operatorname{Re} U(x)(M)>0$ for all $M \in \Re$ and all $x \in B$.

Proof. For each $t \in I, V(x, t)$ satisfies Schwarz' Lemma [17, Theorem A] so $\|V(x, t)\|<\|x\|$ for all $x \in B$. For all $M \in \mathfrak{N}$ and all $x \in B$,

$$
\left|V_{M}(x(M), t)\right|=|V(x, t)(M)| \leqslant\|V(x, t)\| \leqslant\|x\| .
$$

For $z \in \Delta$, the choice $x=z 1$ shows that $V_{M}(\cdot, t)$ satisfies Schwarz' lemma. Now letting $z=x(M)$, we have $|(V(x, t) / x)(M)|=\left|V_{M}(x(M), t) / x(M)\right| \leqslant$ 1 (where the limit value is to be taken when $x(M)=0$ ) and taking the maximum over all $M \in \Re$, we have $\|V(x, t) / x\| \leqslant 1$. Hence,

$$
\operatorname{Re} \frac{x-V(x, t)}{x t}(M) \geqslant \frac{1-\|V(x, t) / x\|}{t} \geqslant 0 \text { for all } t \in I .
$$

The lemma follows.

Definition 2.2. Let $D$ be a domain in $R$. If $U: D \rightarrow R$ is $L$-analytic in $D$ and $\operatorname{Re} U(x)(M) \geqslant 0$ for each $M \in \Re$ and each $x \in D$, then we say $U$ has positive real part in $D$.

EXAMPLE 1. Let $X=\{1,2, \ldots, n\}$ with the discrete topology. Then $C(X)$ $=\mathbf{C}^{n}$ with the multiplication $\left(a_{1}, a_{2}, \ldots, a_{n}\right) \cdot\left(b_{1}, b_{2}, \ldots, b_{n}\right)=$ $\left(a_{1} b_{1}, a_{2} b_{2}, \ldots, a_{n} b_{n}\right)$ and the unit ball $B$ is the polydisk $\left\{\left(z_{1}, z_{2}, \ldots, z_{n}\right)\right.$ : $\left.\left|z_{j}\right|<1,1 \leqslant j \leqslant n\right\}$. Therefore, $L$-analytic functions on $B$ are functions $F\left(z_{1}, z_{2}, \ldots, z_{n}\right)=\left(F_{1}\left(z_{1}\right), F_{2}\left(z_{2}\right), \ldots, F_{n}\left(z_{n}\right)\right)$ where each $F_{j}$ is analytic in the unit disk $\Delta$. There are $n$ maximal ideals $M_{1}, M_{2}, \ldots, M_{n}$ in $C(X)$ given by $M_{j}=\left\{\left(z_{1}, z_{2}, \ldots, z_{n}\right) \in \mathbf{C}^{n}: z_{j}=0\right\}$. It follows that $U=$ $\left(U_{1}, U_{2}, \ldots, U_{n}\right): B \rightarrow \mathbf{C}^{n}$ has positive real part if and only if $\operatorname{Re} U_{j}\left(z_{j}\right)>0$ whenever $\left|z_{j}\right|<1$ (where it is assumed that $U$ is $L$-analytic in $B$ ). Note that if $U$ has positive real part, then $z U$ is in the class $\mathscr{P}$ defined in [15].

EXAMPLE 2. Let $X=[0,1]$ with the usual topology and $R=C(X)$. Then $L$-analytic functions on $B$ are the power series $F(f)=\sum_{n=0}^{\infty} a_{n} f^{n}$ where $a_{n} \in C(X)$ with lim sup $\left\|a_{n}\right\|^{1 / n} \leqslant 1$. The maximal ideals are the sets $M_{x}=$ $\{f \in C(X): f(x)=0\}$ for some $x, 0 \leqslant x \leqslant 1$. Therefore, if $U$ is $L$-analytic 
in $B, U$ has positive real part if and only if $\operatorname{Re} U(f)(x) \geqslant 0$ for all $f \in B$ and $x \in[0,1]$.

EXAMPLE 3. Let $R=H^{\infty}(\Delta)$. In this case, one needs to modify Definition 2.2 to replace $\Re$ by $\Re^{\prime}=\operatorname{cl}\left\{M_{z} \in \mathfrak{R}: f \in M_{z} \Rightarrow \mathrm{f}(\mathrm{z})=0,|z|<1\right\}$. The theory in the remainder of this paper can then be applied to this space. Thus $U: B \rightarrow H^{\infty}(\Delta)$ has positive real part if $\operatorname{Re} U(f)(z) \geqslant 0$ for all $f \in H^{\infty}$ and $z \in \Delta$. For example, $U(f)=(1+f)(1-f)^{-1}=1+2 f+2 f^{2}+\ldots$ has positive real part.

LemMA 2.3. Let $F: B \rightarrow R$ be bianalytic in $B$. Let $G: B \times I \rightarrow R$ be $L$-analytic for each $t \in I, G(x, 0)=F(x)$, for each $x \in B, G(0, t)=F(0)$ for each $t \in I$, and $G(B, t) \subset F(B)$ for each $t \in I$. If $\lim _{t \rightarrow 0^{+}}(G(x, 0)-$ $G(x, t)) / t=x H(x)$ exists and is L-analytic, then $H(x)=F^{\prime}(x) U(x)$ where $U$ has positive real part in $B$.

Proof. We will show that $V(x, t)=F^{-1}(G(x, t))$ satisfies Lemma 2.1. Fix $x \in B, x \neq 0$, and expand $G(x, t)$ about $x$,

$$
G(x, t)=F(V(x, t))=F(x)+F^{\prime}(x)(V(x, t)-x)+K(V(x, t), x)
$$

where $\|K(y, x)\| /\|y-x\| \rightarrow 0$ as $\|y-x\| \rightarrow 0$. Therefore,

$$
\frac{G(x, 0)-G(x, t)}{t}=F^{\prime}(x) \frac{x-V(x, t)}{t}-\frac{K(V(x, t), x)}{t} .
$$

If we show $K(V(x, t), x) / t \rightarrow 0$ as $t \rightarrow 0^{+}$, then

$$
\lim _{t \rightarrow 0^{+}} \frac{x-V(x, t)}{x t}=\left[F^{\prime}(x)\right]^{-1} H(x)
$$

and the lemma follows by Lemma 2.2 .

To show that $K(V(x, t), x) / t \rightarrow 0$ as $t \rightarrow 0^{+}$, observe that $\|(x-$ $V(x, t)) / t \|$ is bounded as $t \rightarrow 0^{+}$; otherwise, for some sequence $\left\{t_{n}\right\}, t_{n} \rightarrow 0$ and $\left\|\left(x-V\left(x, t_{n}\right)\right) / t_{n}\right\| \rightarrow \infty$. In this case,

$$
x H(x)=\lim _{n \rightarrow \infty}\left[F^{\prime}(x) \frac{x-V\left(x, t_{n}\right)}{\left\|x-V\left(x, t_{n}\right)\right\|}-\frac{K\left(V\left(x, t_{n}\right), x\right)}{\left\|x-V\left(x, t_{n}\right)\right\|}\right] \frac{\left\|x-V\left(x, t_{n}\right)\right\|}{t_{n}}
$$

so that

$$
F^{\prime}(x) \frac{x-V\left(x, t_{n}\right)}{\left\|x-V\left(x, t_{n}\right)\right\|} \rightarrow 0 \quad \text { as } n \rightarrow \infty .
$$

But this implies that $F^{\prime}(x)$ is a generalized divisor of zero which contradicts the $L$-analyticity of $F^{-1}$. Thus we have shown

$$
\lim _{t \rightarrow 0^{+}} \frac{K(V(x, t), x)}{t}=\lim _{t \rightarrow 0^{+}} \frac{K(V(x, t), x)}{\|V(x, t)-x\|} \frac{\|V(x, t)-x\|}{t}=0 .
$$


LEMMA 2.4. Let $U$ have positive real part.

(1) If $M \in \mathscr{N}$, then

$$
\begin{array}{r}
\frac{1-\|x\|}{1+\|x\|} \operatorname{Re} U(0)(M) \leqslant \operatorname{Re} U(x)(M) \leqslant \frac{1+\|x\|}{1-\|x\|} \\
\quad \operatorname{Re} U(0)(M) \\
\quad \text { for all } x \in B ;
\end{array}
$$

and so $\operatorname{Re} U(0)(M)>0$ if and only if $\operatorname{Re} U(x)(M)>0$ for all $x \in B$.

(2) $\operatorname{Re} U(0)(M)>0$ for all $M \in \mathfrak{N}$ implies $U(x)$ is nonsingular for all $x \in B$.

Proof. For $M \in \Re$ and $0 \neq x \in B$, let $\rho(\lambda)=U(\lambda x /\|x\|)(M)$ for $\lambda \in$ $\Delta$. Since $\rho$ is holomorphic in $\Delta$ and $\operatorname{Re} \rho(\lambda) \geqslant 0$, by the classical inequality,

$$
\frac{1-|\lambda|}{1+|\lambda|} \operatorname{Re} \rho(0) \leqslant \operatorname{Re} \rho(\lambda) \leqslant \frac{1+|\lambda|}{1-|\lambda|} \operatorname{Re} \rho(0)
$$

so, $\lambda=\|x\|$ yields (1). (2) follows from (1) and the fact that $\operatorname{Re} U(x)(M)>0$ for all $M \in \mathfrak{T}$ implies $U(x) \notin M$ for any $M \in \mathfrak{T}$ and, hence, $U(x)$ is nonsingular.

DEFINITION 2.5. If $U$ has positive real part in a domain $D \subset R$ and Re $U(x)(M)>0$ for all $M \in \mathscr{N}$ and all $x \in D$, then we write $U \in \mathscr{P}(D)$. If $D=B$, then we write $\mathscr{P}$ for $\mathscr{P}(B)$.

Lemma 2.6. Let $P \in \mathscr{P}$. Then, for each $x \in B$, the initial value problem

$$
d w / d t=-w P(w), \quad w(0)=x,
$$

has a unique solution $V(t)=V(x, t)$ defined on $t \geqslant 0$. For fixed $t \geqslant 0$, $V_{t}(x)=V(x, t)$ is L-analytic and univalent in $B$ and

$$
\|V(x, t)\| \leqslant\|x\| \exp \left(-\frac{1-\|x\|}{1+\|x\|} \delta t\right)
$$

for all $t \geqslant 0$ and all $x \in B$ where $\delta=\min \{\operatorname{Re} P(0)(M) \mid M \in \mathfrak{N}\}$.

Proof. The proof of the existence and uniqueness of the solution is covered in [12]. If (1) holds, then the solution can be continued to obtain a solution for all $t \geqslant 0$. The univalence of solution follows from the uniqueness of the solution, and the $L$-analyticity of $V(x, t)$ in $B$ for each $t \geqslant 0$ follows from the equilocal boundedness of the successive approximations $V_{m}(x, t)$ of $V(x, t)$ and Theorem 8.4.3 [6, p. 272].

We now show (1). For each $M \in \mathfrak{T}, V(t)(M)$ is the solution of the initial value problem

$$
d u / d t=-u P_{M}(u), \quad u(0)=V(0)(M)=x(M) .
$$

By [1, Lemma 1], $|V(t)(M)| \leqslant|V(0)(M)|$ for all $t \geqslant 0$. Differentiating $|V(t)(M)|^{2}=V(t)(M) \overline{V(t)(M)}$, we get 


$$
\begin{aligned}
\frac{1}{|V(t)(M)|} \frac{d|V(t)(M)|}{d t} & =-\operatorname{Re} P_{M}(V(t)(M)) \\
& \leqslant-\frac{1-|V(t)(M)|}{1+|V(t)(M)|} \operatorname{Re} P_{M}(0) \\
& \leqslant-\frac{1-|V(0)(M)|}{1+|V(0)(M)|} \operatorname{Re} P_{M}(0) \leqslant-\frac{1-\|x\|}{1+\|x\|} \delta
\end{aligned}
$$

and (1) follows.

3. Starlike functions. In $\mathbf{C}$, if $f(z)=\sum_{n=1}^{\infty} a_{n} z^{n}, a_{1} \neq 0$, is holomorphic in $\Delta$, then $f$ is starlike in $\Delta$ if $(1-t) f(\Delta) \subset f(\Delta)$ for all $t \in I=[0,1]$ which is equivalent to $\operatorname{Re}\left(z f^{\prime}(z) / f(z)\right)>0$ for all $z \in \Delta$. We will define starlike functions in $R$ and relate them to starlike function in $\mathbf{C}$.

Definition 3.1. A bianalytic map $F: B \rightarrow R$ is said to be starlike in $B$ if $F(0)=0$ and $(1-t) F(B) \subset F(B)$ for all $t \in I$.

THeOREM 3.2. Let $F(x)=\sum_{n=1}^{\infty} a_{n} x^{n}$ be locally bianalytic in $B$. Then $F$ is starlike in $B$ if and only if $F_{M}(z)=\sum_{n=1}^{\infty} a_{n}(M) z^{n}$ is starlike in $\Delta$ for all $M \in \mathfrak{R}$.

Proof. Assume $F$ is starlike in $B$ and set $G(x, t)=(1-t) F(x)$. Lemma 2.3 applies with $x H(x)=F(x)$ so that $F(x)=x F^{\prime}(x) U(x)$ where $U$ has positive real part. However, $U(0)=1$ by equating coefficients, so by Lemma 2.4, $U \in \mathscr{P}$. Setting $x=z e$, we conclude

$$
\operatorname{Re} \frac{\sum_{n=1}^{\infty} a_{n}(M) z^{n-1}}{\sum_{n=1}^{\infty} n a_{n}(M) z^{n-1}}>0 \quad \text { for } z \in \Delta
$$

and, hence, $F_{M}$ is starlike for each $M \in \mathfrak{N}$.

Conversely, if $F_{M}$ is starlike for every $M \in \mathfrak{R}$, then for fixed $x \in B$, the function $V(x, t)=F^{-1}\left(e^{-t} F(x)\right)$, defined near $t=0$, satisfies the initial value problem

$$
\frac{\partial V(x, t)}{\partial t}=-\left[\frac{F(V(x, t))}{V(x, t) F^{\prime}(V(x, t))}\right] V(x, t), \quad V(x, 0)=x .
$$

Set $P(w)=F(w) / w F^{\prime}(w)$ for all $w \in B$. By hypothesis, $P \in \mathcal{P}$ and so by Lemma 2.6, $V(x, t)$ is the unique solution of the initial value problem

$$
d w / d t=-w P(w), \quad w(0)=x .
$$

Then $\|V(x, t)\| \leqslant\|x\|<1$ and $F(V(x, t))=e^{-t} F(x), t>0$. This implies that $(1-t) F(B) \subset F(B), 0 \leqslant t \leqslant 1$. To see the univalence of $F$ in $B$, let $x_{1}, x_{2} \in B$ such that $F\left(x_{1}\right)=F\left(x_{2}\right)$. Suppose $V_{x_{i}}(t)=V\left(x_{i}, t\right)$ is the unique solution of 


$$
d w / d t=-w P(w), \quad w(0)=x_{i},
$$

and let $W_{x_{i}}(t)=F\left(V_{x_{i}}(t)\right), i=1,2$. For small $t \geqslant 0, W_{x_{i}}(t)$ satisfies the initial value problem

$$
d w / d t=-w, \quad w(0)=F\left(x_{i}\right),
$$

which has a unique solution $W_{x_{i}}(t)=F\left(x_{i}\right) e^{-t}$ for $t>0$. Since $F\left(x_{1}\right)=$ $F\left(x_{2}\right), W_{x_{1}}(t)=W_{x_{2}}(t)$ for $t \geqslant 0$. Since $W_{x_{i}}(t) \rightarrow 0$ as $t \rightarrow+\infty$, and since $F$ has a local inverse in an open neighborhood of $0, V_{x_{1}}(t)=V_{x_{2}}(t)$ for all $t \geqslant M>0$. Then $V_{x_{1}}(t)=V_{x_{2}}(t)$ for all $t \geqslant 0$; in particular, $x_{1}=V_{x_{1}}(0)=$ $V_{x_{2}}(0)=x_{2}$ and $F$ is univalent in $B$.

EXAMPLE 1. Let $F: B \rightarrow R$ be given by $F(x)=x(1-a x)^{-2}$ where $\|a\|<$ 1. Let $M \in \mathfrak{T}$ and set $a(M)=\alpha$. Then $|\alpha| \leqslant 1$ and $F_{M}(z)=z /(1-\alpha z)^{2}$, which is known to be starlike. Therefore $F$ is starlike. If $X=\{1,2, \ldots, n\}$ so that $C(X)=\mathrm{C}^{n}=R, F$ has the form

$$
\begin{array}{r}
F\left(z_{1}, z_{2}, \ldots, z_{n}\right)=\left(z_{1} /\left(1-a_{1} z\right)^{2}, \ldots, z_{n} /\left(1-a_{n} z\right)^{2}\right) \\
\text { where }\left|a_{j}\right|<1,1 \leqslant j \leqslant n .
\end{array}
$$

If $R=C[0,1]$,

$$
F(f)(x)=f(x) /(1-a(x) f(x))^{2}, \quad 0 \leqslant x \leqslant 1 .
$$

If $R=H^{\infty}(\Delta)$,

$$
F(f)(z)=f(z) /(1-a(z) f(z))^{2}, \quad|z|<1 .
$$

EXAMPLE 2. Other choices for $F: B \rightarrow R$ that will make $F$ starlike are

$$
\begin{gathered}
F(x)=x+a x^{2}, \quad a \in R,\|a\| \leqslant \frac{1}{2}, \\
F(x)=x \prod_{j=1}^{\infty}\left(1-a_{j} x\right)^{-\alpha_{j}}
\end{gathered}
$$

where each $\alpha_{j} \geqslant 0$ and $\sum_{j=1}^{\infty} \alpha_{j} \leqslant 2$ with $\left\|a_{j}\right\| \leqslant 1$ for each $j$.

4. Convex functions. In $\mathbf{C}$, if $f(z)=\sum_{n=1}^{\infty} a_{n} z^{n}, a_{1} \neq 0$, is holomorphic in $\Delta$, then $f$ is convex in $\Delta$ if $f(\Delta)$ is a convex domain. This is equivalent to $\operatorname{Re}\left(1+z f^{\prime \prime}(z) / f^{\prime}(z)\right)>0$ for all $z \in \Delta$. We will define convex functions in $R$ and relate them to convex functions in $\mathbf{C}$.

Defintion 4.1. A bianalytic map $F: B \rightarrow R$ is said to be convex in $B$ if $F(B)$ is a convex domain.

THEOREM 4.2. Let $F(x)=\sum_{n=1}^{\infty} a_{n} x^{n}$ be locally bianalytic in $B . F$ is convex in $B$ if and only if $F_{M}(z)=\sum_{n=1}^{\infty} a_{n}(M) z^{n}$ is convex in $\Delta$ for each $M \in \Re$. Thus the Alexander relation ( $F$ is convex in $B$ if and only if $G$ is starlike in $B$ where $G(x)=x F^{\prime}(x)$ for all $\left.x \in B\right)$ holds. 
Proof. Assume $F$ is convex in $B$. Set $G(x, t)=\frac{1}{2}\left(F\left(e^{i{ }^{t}} x\right)+F\left(e^{-i \sqrt{ } t} x\right)\right)$ and apply Lemma 2.3. Expanding $F\left(e^{ \pm i \sqrt{ } t} x\right)$ about $x$, we have

$$
\begin{aligned}
& \frac{1}{2}\left(F\left(e^{i \bigvee t} x\right)+F\left(e^{-i \bigvee t} x\right)\right) \\
& =\frac{1}{2}\left[F(x)+F^{\prime}(x)\left(e^{i \sqrt{ } t}-1\right) x+\frac{1}{2} F^{\prime \prime}(x)\left(e^{i \sqrt{ } t}-1\right)^{2} x^{2}\right. \\
& \left.+F(x)+F^{\prime}(x)\left(e^{-i \sqrt{ } t}-1\right) x+\frac{1}{2} F^{\prime \prime}(x)\left(e^{-i \sqrt{ } t}-1\right)^{2} x^{2}+o(t)\right] \\
& =F(x)+x F^{\prime}(x)(\cos \bigvee t-1) \\
& +\frac{1}{2} x^{2} F^{\prime \prime}(x)(\cos 2 \sqrt{ } t-2 \cos \bigvee t+1)+o(t) \text {. }
\end{aligned}
$$

Therefore,

$$
\begin{aligned}
\lim _{t \rightarrow 0^{+}} \frac{G(x, 0)-G(x, t)}{t} & \\
= & x F^{\prime}(x) \lim _{t \rightarrow 0^{+}} \frac{1-\cos \bigvee t}{t}+x^{2} F^{\prime \prime}(x) \lim _{t \rightarrow 0^{+}} \cos \sqrt{ } t\left(\frac{(1-\cos V t)}{t}\right) \\
& =\frac{1}{2}\left[x F^{\prime}(x)+x^{2} F^{\prime \prime}(x)\right] .
\end{aligned}
$$

Therefore $F^{\prime}(x) U(x)=\frac{1}{2}\left[F^{\prime}(x)+x F^{\prime \prime}(x)\right]$ where $U$ has positive real part. Equating coefficients, we conclude that $U(0)=\frac{1}{2} 1$ and so $U \in \mathcal{P}$. This means

$$
\operatorname{Re}\left[1+\frac{\sum_{n=1}^{\infty} n^{2} a_{n}(M) z^{n-1}}{\sum_{n=1}^{\infty} n a_{n}(M) z^{n-1}}\right]>0 \quad \text { for all } M \in \Re
$$

so that $F_{M}$ is convex in $\Delta$.

Suppose $F_{M}$ is convex in $\Delta$ for each $M \in \mathscr{T}$ and let $x, y \in B_{r}=\{x \in$ $R \mid\|x\|<r\}, r<1$. Since $F_{M}$ is univalent, $F$ is bianalytic in $B$. Let $V(t)=$ $F^{-1}(t F(x)+(1-t) F(y))$. Then for all $M \in \mathfrak{N}$,

$$
F_{M}(V(t)(M))=t F_{M}(x(M))+(1-t) F_{M}(y(M)) \text {. }
$$

Since $F_{M}$ is convex in $|z|<r,|V(t)(M)|<r$. Choose $M \in \mathfrak{N}$ such that $\|V(t)\|=|V(t)(M)|<r$ and the convexity of $F$ follows.

EXAMPLE. (i) $x(1-a x)^{-1}$ when $\|a\| \leqslant 1$ is convex.

(ii) $\log \left[(1+x)(1-x)^{-1}\right]$ is convex.

5. Close-to-convex functions. In $\mathbf{C}$, a holomorphic function $f: \Delta \rightarrow \mathbf{C}$ is said to be close-to-convex in $\Delta$ if there is a convex function $g: \Delta \rightarrow C$ such that $\operatorname{Re}\left(f^{\prime}(z) / g^{\prime}(z)\right)>0$ for all $z \in \Delta$. In [9], it is shown that every close-toconvex function is univalent. We define close-to-convex functions in $R$ and show that every close-to-convex function in $B$ is univalent. Compare [13] and [17]. 
Definition 5.1. Suppose $F: B \rightarrow R$ is $L$-analytic in $B$. We say that $F$ is close-to-convex if $F_{M}: \Delta \rightarrow \mathrm{C}$ is close-to-convex in $\Delta$ for all $M \in \mathfrak{N}$.

Clearly if $G: B \rightarrow R$ is convex in $B, U \in \mathscr{P}$, and $F^{\prime}(x)=G^{\prime}(x) U(x)$ for all $x \in B$, then $F$ is close-to-convex in $B$.

THEOREM 5.2. If $D$ is a convex domain in $R$ and $G: D \rightarrow R$ is such that $G^{\prime} \in \mathcal{P}(D)$, then $G$ is univalent in $D$.

Proof. Let $x_{1}, x_{2} \in D, x_{1} \neq x_{2}$. Since $D$ is convex, $\left\{t x_{2}+(1-t) x_{1} \mid t \in\right.$ I\} $\subset D$. We have

$$
\frac{d}{d t} G\left(t x_{2}+(1-t) x_{1}\right)=G^{\prime}\left(t x_{2}+(1-t) x_{1}\right)\left(x_{2}-x_{1}\right)
$$

so that

$$
G\left(x_{2}\right)-G\left(x_{1}\right)=\left(x_{2}-x_{1}\right) \int_{0}^{1} G^{\prime}\left(t x_{2}+(1-t) x_{1}\right) d t
$$

Let $M \in \mathscr{N}$ be such that $\left\|x_{2}-x_{1}\right\|=\left|\left(x_{2}-x_{1}\right)(M)\right|$. Then

$$
\begin{aligned}
\left|\left(G\left(x_{2}\right)-G\left(x_{1}\right)\right)(M)\right| & =\left\|x_{2}-x_{1}\right\|\left|\int_{0}^{1} G^{\prime}\left(t x_{2}+(1-t) x_{1}\right)(M) d t\right| \\
& \geqslant\left\|x_{2}-x_{1}\right\| \int_{0}^{1} \operatorname{Re} G^{\prime}\left(t x_{2}+(1-t) x_{1}\right)(M) d t>0
\end{aligned}
$$

and hence $G\left(x_{2}\right) \neq G\left(x_{1}\right)$.

THEOREM 5.3. If $F$ is close-to-convex in $B$, then $F$ is univalent in $B$.

Proof. If there is a convex function $G: B \rightarrow R$ such that $F^{\prime}(x)=$ $G^{\prime}(x) U(x)$ for some $U \in \mathcal{P}$, we may apply Theorem 5.2 to $F \circ G^{-1}$ : $G(B) \rightarrow R$ to conclude that $F$ is univalent.

Otherwise, let $x_{1}, x_{2} \in B, x_{1} \neq x_{2}$ and choose $M \in \Re$ such that $\mid\left(x_{2}-\right.$ $\left.x_{1}\right)(M) \mid=\left\|x_{2}-x_{1}\right\|$. Since $F_{M}$ is close-to-convex in $\Delta$, there is a convex function $g: \Delta \rightarrow \mathrm{C}$ such that $\operatorname{Re}\left(F_{M}^{\prime}(z) / g^{\prime}(z)\right)>0$ for all $z \in \Delta$. Define $G$ : $B \rightarrow R$ by $G(x)=\sum_{k=1}^{\infty}\left(b_{k} 1\right) x^{k}$ where $g(z)=\sum_{k=1}^{\infty} b_{k} z^{k}$. Then $G$ is convex (in particular, bianalytic) in $B$. Consider $H \equiv F \circ G^{-1}: G(B) \rightarrow R$ and let $y_{1}=G\left(x_{1}\right)$ and $y_{2}=G\left(x_{2}\right)$. As in the proof of Theorem 5.2, we have

$$
F\left(x_{2}\right)-F\left(x_{1}\right)=H\left(y_{2}\right)-H\left(y_{1}\right)=\int_{0}^{1} H^{\prime}\left(t y_{2}+(1-t) y_{1}\right)\left(y_{2}-y_{1}\right) d t
$$


so that

$$
\begin{aligned}
\left|\left(F\left(x_{2}\right)-F\left(x_{1}\right)\right)(M)\right|=\left|\left(H\left(y_{2}\right)-H\left(y_{1}\right)\right)(M)\right| \\
\quad=\left|\int_{0}^{1} H^{\prime}\left(t y_{2}+(1-t) y_{1}\right)(M) d t\right|\left|\left(y_{2}-y_{1}\right)(M)\right| \\
>\int_{0}^{1} \operatorname{Re} H^{\prime}\left(t y_{2}+(1-t) y_{1}\right)(M) d t\left|\left(y_{2}-y_{1}\right)(M)\right| \\
\quad=\int_{0}^{1} \operatorname{Re} \frac{F_{M}^{\prime}\left(G^{-1}\left(t y_{2}+(1-t) y_{1}\right)(M)\right)}{g^{\prime}\left(G^{-1}\left(t y_{2}+(1-t) y_{1}\right)(M)\right)} d t\left|\left(y_{1}-y_{2}\right)(M)\right|>0
\end{aligned}
$$

if $\left|\left(y_{2}-y_{1}\right)(M)\right| \neq 0$. But

$$
\begin{aligned}
\left\|x_{2}-x_{1}\right\| & =\left|\left(x_{2}-x_{1}\right)(M)\right| \\
& =\left|\int_{0}^{1}\left(G^{-1}\right)^{\prime}\left(t y_{2}+(1-t) y_{1}\right)(M) d t\right|\left|\left(y_{2}-y_{1}\right)(M)\right|
\end{aligned}
$$

so the desired result follows.

EXAMPLE. (i) $F(x)=x(1-a x)(1-x)^{-2}$ is close-to-convex in $B$ where $\left\|a-\frac{1}{2} 1\right\|<\frac{1}{2}$ because $F_{M}(z)$ is known to be close-to-convex for every $M \in$ গ⿰.

(ii) Every starlike function is close-to-convex.

(iii) Every convex function is close-to-convex.

6. Spirallike functions. In $\mathbf{C}$, if $f(z)=\sum_{n=1}^{\infty} a_{n} z^{n}, a_{1} \neq 0$, is holomorphic in $\Delta$, then $f$ is spirallike in $\Delta$ if $\operatorname{Re}\left(e^{-i \alpha} z f^{\prime}(z) / f(z)\right)>0$ for all $z \in \Delta$ where $\alpha \in(-\pi / 2, \pi / 2)$. If $f$ is spirallike in $\Delta$, then $f$ is univalent in $\Delta$ [14]. We will define spirallike functions in $R$ and prove that they are also univalent in $B$.

Definition 6.1. Suppose $F(x)=\sum_{n=1}^{\infty} a_{n} x^{n}$ is locally bianalytic in $B$. We say that $F$ is spirallike in $B$ if there exists $a \in R$ such that $\operatorname{Re} a(M)>0$ for all $M \in \mathfrak{N}$ and $U \in \mathscr{P}$ such that

$$
a \frac{F(x)}{x}=F^{\prime}(x) U(x) \text { for all } x \in B\left(\text { where } \frac{F(x)}{x}=\sum_{n=1}^{\infty} a_{n} x^{n-1}\right) \text {. }
$$

From (1), we see that $(F(x) / x)(M) \neq 0$ whenever $M \in \Re$ and so $F(x) / x$ and $a$ are nonsingular. It is clear that (1) can be replaced by the condition $\operatorname{Re}\left(b x F^{\prime}(x) / F(x)\right)(M)>0$ for all $M \in \mathscr{T}$ where $b=a^{-1}$ and $x F^{\prime}(x) / F(x)$ means $(F(x) / x)^{-1} F^{\prime}(x)$.

THEOREM 6.2. Every spirallike function in $B$ is univalent in B. Furthermore, if $F$ is spirallike in $B$, then $F_{M}$ is spirallike in $\Delta$ for each $M \in \mathfrak{R}$. 
Proof. Since $F^{\prime}(x)$ is nonsingular for each $x \in B, F$ is locally bianalytic in $B$. For fixed $x \in B$ and $t$ near zero, set $V(x, t)=F^{-1}\left(e^{-a t} F(x)\right)$. Then $V(x, t)$ is a solution of the initial value problem

$$
d w / d t=-w P(w), \quad w(0)=x,
$$

where $P(w)=a F(w) / w F^{\prime}(w)$. By (1), $P \in \mathscr{P}$ and so by Lemma 2.6, $V(x, t)$ is the unique solution for $t \geqslant 0$ and $V(x, t) \rightarrow 0$ as $t \rightarrow \infty$. Let $x_{1}, x_{2} \in B$ such that $F\left(x_{1}\right)=F\left(x_{2}\right)$ and let $V_{x_{i}}(t)=V\left(x_{i}, t\right)$ be the unique solution of the initial value problem

$$
d w / d t=-w P(w), \quad w(0)=x_{i}, i=1,2 .
$$

Let $W_{x_{i}}(t)=F\left(V_{x_{i}}(t)\right)$ for all $t \geqslant 0, i=1,2$. For small $t \geqslant 0, W_{x_{i}}(t)$ satisfies the initial value problem

$$
d w / d t=-a w, \quad w(0)=F\left(x_{i}\right),
$$

which has a unique solution $W_{x_{i}}(t)=F\left(x_{i}\right) e^{-a t}$ for $t \geqslant 0$. Since $F\left(x_{1}\right)=$ $F\left(x_{2}\right), W_{x_{1}}(t)=W_{x_{2}}(t)$ for all $t>0$. Since $W_{x_{i}}(t) \rightarrow 0$ as $t \rightarrow+\infty$, we conclude that $x_{1}=x_{2}$ as in Theorem 3.2.

That $F_{M}$ is spirallike in $\Delta$ follows from the equation $a(M)[F(x) / x](M)=$ $F^{\prime}(x)(M) U(x)(M)$, and writing $x=z 1$ gives $a(M)\left[F_{M}(z) / z\right]=F_{M}^{\prime}(z) U_{M}(z)$ where $\operatorname{Re} U_{M}(z)>0$. Write $a(M)=|a(M)| e^{i \alpha}$ where $\alpha \in(-\pi / 2, \pi / 2)$.

EXAMPLE. Let

$$
F(x)=x(1-a x)^{-(1+b)}=\sum_{n=1}^{\infty} \frac{(b+1)(b+2 \cdot 1) \cdots(b+n \cdot 1)}{n} a^{n} x^{n}
$$

where $\|a\|<1,\|b\|<1$ and $-1<\operatorname{Re} b(M)<1$ for all $M \in \mathfrak{R}$. For example, one might take $b=\rho e^{i \alpha} \cdot 1$ where $\alpha$ is real, $0<|\alpha|<\pi$ and $0<\rho<1$. Then

$$
(F(x) / x) \cdot\left(F^{\prime}(x)\right)^{-1} \equiv(1-a x)(1+a b x)^{-1},
$$

and setting $U(x)=(1+b)(F(x) / x)\left(F^{\prime}(x)\right)^{-1}$ yields

$\operatorname{Re} U(x)(M)$

$$
=\operatorname{Re}\left[(1+b(M))(1-a(M) x(M))(1+a(M) b(M) x(M))^{-1}\right] .
$$

With $z=a(M) x(M)$ and $\beta=b(M)$,

$$
U(x)(M)=(1+\beta)(1-z) /(1+\beta z),
$$

and it is easy to show $\operatorname{Re} U(x)(M)>0$.

7. $\Phi$-like functions. See [1] for the definitions of a $\Phi$-like function and a $\Phi$-like domain in $\mathbf{C}$.

Definition 7.1. Let $F: B \rightarrow R$ be a locally bianalytic function in $B$, and $F(0)=0$. If $\Phi: F(B) \rightarrow R$ is $L$-analytic, then we say $F$ is $\Phi$-like in $B$ if there is $U \in \mathcal{P}$ such that $\Phi(F(x))=x F^{\prime}(x) U(x)$ for all $x \in B$. 
Since $F(0)=0, \Phi(0)=0$. Letting $\alpha \rightarrow 0$ in $\Phi(F(\alpha x)) / \alpha=x F^{\prime}(\alpha x) U(\alpha x)$, we have $\Phi^{\prime}(0) x=x U(0)$ for all $x \in B$. Setting $x=\alpha 1$, where $0<|\alpha|<1$, we see that $\Phi^{\prime}(0)=U(0)$.

Definition 7.2. Let $D$ be a domain in $R$ which contains 0 and let $P \in \mathcal{P}(D)$. If, for each $\alpha \in D$, the initial value problem

$$
d w / d t=-\Phi(w), \quad w(0)=\alpha,
$$

where $\Phi(w)=w P(w)$, has a unique solution $w=W(t) \in D$ for all $t>0$ and $W(t) \rightarrow 0$ as $t \rightarrow+\infty$, then $D$ is said to be $\Phi$-like.

Note that starlike, convex, close-to-convex and spirallike functions are $\Phi$-like for appropriate choices of $\Phi$.

TheOREM 7.3. If $F$ is $\Phi$-like in $B$ for $\Phi(v)=v P(v)$ where $P \in \mathscr{P}(F(B)$ ), then $F$ is univalent in $B$ and $F(B)$ is $\Phi$-like.

Proof. The proof follows along the lines of the proof of Theorem 1 in [5].

THEOREM 7.4. If $F: B \rightarrow R$ is bianalytic in $B$ with $F(0)=0$ and $F(B)$ is $\Phi$-like, then $F$ is $\Phi$-like in $B$.

Since our proof uses Lemma 2.3, and therefore is shorter than Theorem 2 [5], we will give our proof.

Proof. Since $F(B)$ is $\Phi$-like, for each $x \in B$, let $W_{x}(t)$ be the unique solution of $d w / d t=-\Phi(w), w(0)=F(x)$ where $\Phi(w)=w P(w), \quad P \in$ $\mathscr{P}(F(B))$. Since $F$ is bianalytic in $B$, set $V_{x}(t)=F^{-1}\left(W_{x}(t)\right)$ for all $t \geqslant 0$. Then $V_{x}(0)=x$ and

$$
F^{\prime}\left(V_{x}(t)\right) V_{x}^{\prime}(t)=W_{x}^{\prime}(t)=-W_{x}(t) P\left(W_{x}(t)\right)=-F\left(V_{x}(t)\right) P\left(F\left(V_{x}(t)\right)\right)
$$

for all $t \geqslant 0$. Letting $t=0$, we have $-F^{\prime}(x) V_{x}^{\prime}(0)=\Phi(F(x))$. To show that $-V_{x}^{\prime}(0)=x U(x)$ for some $U \in \mathcal{P}$, let $G(x, t)=W_{x}(t)=F\left(V_{x}(t)\right)$ in Lemma 3.2. Then

$$
\begin{aligned}
\lim _{t \rightarrow 0^{+}} \frac{G(x, 0)-G(x, t)}{t} & =\lim _{t \rightarrow 0^{+}} \frac{F\left(V_{x}(0)\right)-F\left(V_{x}(t)\right)}{t} \\
& =-F^{\prime}(x) V_{x}^{\prime}(0)=x H(x)
\end{aligned}
$$

is $L$-analytic in $B$ and so $H(x)=F^{\prime}(x) U(x)$ where $U$ has positive real part. Hence $x U(x)=-V_{x}^{\prime}(0)$. Since $x F^{\prime}(x) U(x)=\Phi(F(x))$, we have $U(0)=$ $\Phi^{\prime}(0)=P(0)$, and so $\operatorname{Re} U(0)(M)=\operatorname{Re} P(0)(M)>0$ for all $M \in \Re$ and, by Lemma $2.4, U \in \mathcal{P}$.

8. Convex $F$-holomorphic functions in $C(X)$. If $D$ is an open set in the Banach space $\mathscr{B}$ and $F: D \rightarrow \mathscr{B}$, then $F$ is said to be $F$-holomorphic in $D$ if for each $x \in D$, there is a bounded linear map $D F(x): \mathscr{B} \rightarrow \mathscr{B}$ such that $\lim _{h \rightarrow 0}\|F(x+h)-F(x)-D F(x)(h)\| /\|h\|=0$. If $F: D \rightarrow \mathscr{B}$ is $F$-holomorphic in $D$ and for each $y \in F(B)$, there is an open neighborhood $V$ of $y$ 
such that $F^{-1}: V \rightarrow D$ is $F$-holomorphic in $V$, then we say $F$ is locally biholomorphic in $B$. If $F$ is univalent and locally biholomorphic in $B$, we say that $F$ is biholomorphic in $B$. If $F$ is $F$-holomorphic in $D$, then for each $x_{0} \in D$ there is a disk in $D$ with center at $x_{0}$ such that $F(x)=$ $\sum_{n=0}^{\infty} 1 / n ! D^{n} F\left(x_{0}\right)\left(\left(x-x_{0}\right)^{n}\right)$ where $D^{n} F\left(x_{0}\right) \in L_{n}(\mathscr{B}, \mathscr{B})$, space of all continuous symmetric $n$-linear maps of $\mathscr{B}^{n}$ into $\mathscr{B}$ and the series converges uniformly in this disk. If $F: B \rightarrow \mathscr{B}$ is $F$-holomorphic in $B$, then $F(x)=$ $\sum_{n=0}^{\infty} 1 / n ! D^{n} F(0)\left(x^{n}\right)$ for all $x \in B$ [7, Theorem 3.16.2]. If $\mathscr{B}$ is a commutative Banach algebra with identity, it is clear that every $L$-analytic function is $F$-holomorphic; however, not every $F$-holomorphic function is $L$-analytic [7, p. 115]. We will prove that $F$-holomorphic implies $L$-analytic in the special case in which $F$ is a biholomorphic map of the unit ball of $C(X)$ onto a convex domain in $C(X)$.

Assume $X$ is a compact $T_{2}$-space such that each point of $X$ is a $G_{\delta}$; i.e., each $x \in X$ is the intersection of a countable number of open neighborhoods of $x$. Let $C(X)$ be the Banach algebra of complex valued continuous functions on $X$ (with sup norm and pointwise multiplication).

THEOREM 8.1. Let $C(X)$ be as above. If $F: B \rightarrow C(X)$ is a convex biholomorphic function in $B$ such that $D F(0)=I$, then $F$ is $L$-analytic in $B$ and hence bianalytic in $B$.

Without loss of generality, we assume $F(0)=0$. The proof will be given in the following six lemmas.

LEMMA 8.2. If $k, u \in C(X), k \equiv 0$ on an open neighborhood of $x_{0} \in X$, $u\left(x_{0}\right)=1$ and $|u(x)|<1$ if $x \neq x_{0}$ (such a peaking function $u$ exists since every point of $X$ is $\left.G_{\delta}\right)$, then when $|\alpha|<1$, we have

$$
[D F(\alpha u)]^{-1}\left(D^{n} F(\alpha u)\left(k^{n}\right)\right)\left(x_{0}\right)=0 \text { for } n=2,3, \ldots
$$

Proof. Assume $k \equiv 0$ on the open neighborhood $N$ of $x_{0}$. Then $N^{c}$, complement of $N$, is compact, so that for fixed $\alpha, 0<|\alpha|<1$, we can choose $r>0$ (say $r=|\alpha|(1-m) /(\|k\|+1)$ where $m=\sup \left\{|u(x)| \mid x \in N^{c}\right\}<1$ ) so that

$$
\|\alpha u+\beta k\|=\left|(\alpha u+\beta k)\left(x_{0}\right)\right|=|\alpha| \text { for all } \beta \in \mathbf{C},|\beta|<r .
$$

Define $l \in C(X)^{*}$ by $l(f)=|\alpha| f\left(x_{0}\right) / \alpha$ for all $f \in C(X)$. Then $l(\alpha u)=$ $|\alpha|=\|\alpha u\|$ and $\|l\|=1$. Since $F$ is convex biholomorphic in $B$, we know by Theorem 4 [16, p. 583] there is a function $w: B \times B \rightarrow C(X)$ such that $w$ is $F$-holomorphic in each variable, $w(\alpha u, \alpha u)=0, \operatorname{Re} l(w(\alpha u, \alpha u+\beta k))>0$ if $|\beta|<r$, and

$$
F(\alpha u)-F(\alpha u+\beta k)=D F(\alpha u)(w(\alpha u, \alpha u+\beta k)) .
$$


Expanding $F(\alpha u+\beta k)$ about $\alpha u$, we have

$$
F(\alpha u+\beta k)=F(\alpha u)+\sum_{n=1}^{\infty} \frac{\beta^{n}}{n !} D^{n} F(\alpha u)\left(k^{n}\right)
$$

so that

$$
w(\alpha u, \alpha u+\beta k)=-\beta k-\sum_{n=2}^{\infty} \frac{\beta^{n}}{n !}[D F(\alpha u)]^{-1} D^{n} F(\alpha u)\left(k^{n}\right) .
$$

Applying $l$, we have

$$
v(\beta) \equiv l w(\alpha u, \alpha u+\beta k)=-\sum_{n=1}^{\infty} \frac{\beta^{n}}{n !} l\left([D F(\alpha u)]^{-1} D^{n} F(\alpha u)\left(k^{n}\right)\right)
$$

is a holomorphic function of $\beta$ for $|\beta|<r$ and $\operatorname{Re} v(\beta) \geqslant 0$. Since $v(0)=0$, $v(\beta) \equiv 0$ and Lemma 8.2 follows.

Lemma 8.3. If $k, u \in C(X), k\left(x_{0}\right)=0$ and $u$ is as in Lemma 8.2, then $[D F(\alpha u)]^{-1}\left(D^{n} F(\alpha u)\left(k^{n}\right)\right)\left(x_{0}\right)=0$ for $n=2,3, \ldots$

We will prove this without utilizing the $G_{\delta}$ property. Let $A$ be an index set of the open neighborhoods of $x_{0}$ and let $A_{\alpha}$ be the set of functions $u_{8} k$ where $u_{\delta} \in C(X), u_{\delta}=1$ on $U_{\alpha}^{c}$, complement of $U_{\alpha}$, support of $u_{\delta} \subset \bar{U}_{\delta}^{c}, 0<u_{\delta}<$ 1 , where $U_{\delta}$ is an open neighborhood of $x_{0}$ and $\bar{U}_{\delta} \subset U_{\alpha} . A_{\alpha} \neq \varnothing$ by Urysohn's Lemma. Let $\mathcal{Q}=\left\{A_{\alpha} \mid \alpha \in A\right\}$. It is routine to show that $\mathcal{Q}$ is a filterbase in $C(X)$ which converges (in the norm topology) to $k$ [2, p. 211]. Apply Lemma 8.2 to each $u_{\delta} k$ and the result follows.

LEMMA 8.4. If $k$, $u$ are as in Lemma 8.3, we have

$$
[D F(\alpha u)]^{-1} D^{2} F(\alpha u)(u, k)\left(x_{0}\right)=0 .
$$

Proof. First assume $k$ is as in Lemma 8.2. Set $\left(1+t^{2}\right)^{1 / 2} g=\alpha(1+i t) u+$ $\beta k$ where $|\beta|=t^{1 / 2}$, and $t$ is sufficiently small and positive so that

$$
\|g\|=\left|\frac{\alpha(1+i t) u\left(x_{0}\right)+\beta k\left(x_{0}\right)}{\sqrt{1+t^{2}}}\right|<|\alpha|<1 .
$$

Let $l$ be the same as in the proof of Lemma 8.2. Since $F$ is convex biholomorphic in $B$, we know again by Theorem 4 [16] that $F(\alpha u)-F(g)=$ $D F(\alpha u)(w(\alpha u, g))$ where $\operatorname{Re} l(w(\alpha u, g)) \geqslant 0$. Expanding $F(g)$ about $\alpha u$, we have

$$
\begin{aligned}
F(g)= & F(\alpha u)+\sum_{n=1}^{\infty} \frac{1}{n !} D^{n}(\alpha u)(g-\alpha u)^{n} \\
= & F(\alpha u)+D F(\alpha u)(g-\alpha u) \\
& +D^{2} F(\alpha u)\left(\frac{i+\alpha u}{\sqrt{1+t^{2}}}, \frac{\beta k}{\sqrt{1+t^{2}}}\right)+O\left(t^{2}\right)
\end{aligned}
$$


since $\left(1+t^{2}\right)^{-1 / 2}-1=O\left(t^{2}\right)$ and $|\beta|=t^{1 / 2}$. By Lemma 8.2 we then have

$$
w(\alpha u, g)=-(g-\alpha u)-\frac{i \alpha u t \beta}{1+t^{2}}[D F(\alpha u)]^{-1} D^{2} F(\alpha u)(u, k)+O\left(t^{2}\right)
$$

so that

$$
\begin{aligned}
\frac{|\alpha|}{\alpha} w(\alpha u, g)\left(x_{0}\right)= & \frac{-i t|\alpha|}{\sqrt{1+t^{2}}} \\
& +\frac{-i t|\alpha| \beta}{1+t^{2}}[D F(\alpha u)]^{-1} D^{2} F(\alpha u)(u, k)\left(x_{0}\right)+O\left(t^{2}\right)
\end{aligned}
$$

Since $\operatorname{Re} l(w(\alpha u, g)) \geqslant 0$, we conclude that

$$
\operatorname{Re} \frac{-i|\alpha| t \beta}{1+t^{2}}[D F(\alpha u)]^{-1} D^{2} F(\alpha u)(u, k)\left(x_{0}\right)>0 .
$$

Since $\arg \beta$ is arbitrary, we have

$$
[D F(\alpha u)]^{-1} D^{2} F(\alpha u)(u, k)\left(x_{0}\right)=0 \text { if } k \equiv 0
$$

in an open neighborhood of $x_{0}$. Now assume $k \in C(X)$ and $k\left(x_{0}\right)=0$. Apply the argument in Lemma 8.3 to obtain the conclusion.

LEMMA 8.5. If $k, u$ are as in Lemma 8.3, then

$$
[D F(\alpha u)]^{-1} D^{n} F(\alpha u)\left(u^{l}, k^{n-l}\right)\left(x_{0}\right)=0
$$

when $0<l<n, n=2,3,4, \ldots$

Proof. When $l=0$ and $n \geqslant 2$, the result is Lemma 8.3. When $l=1$ and $n=2$, the result is Lemma 8.4. Assume the result is true for fixed $l$ and $n$ and prove it for $l+1$ and $n+1$. Let $G=F^{-1}$. Then, for all $f \in B$, we have $G \circ F(f)=f, D G(F(f)) \circ D F(f)=I$, identity map from $C(X)$ into $C(X)$, and

$$
D^{2} G(F(f))(D F(f)(g), D F(f)(h))+D G(F(f))\left(D^{2} F(f)(g, h)\right)=0,
$$

zero map from $C(X)$ into $C(X)$, for all $f \in B$ and all $g, h \in C(X)$. Hence

$$
\begin{aligned}
D^{2} G(F(f)) & (g, h) \\
= & -D G(F(f))\left[D^{2} F(f)\left([D F(f)]^{-1}(g),[D F(f)]^{-1}(h)\right)\right] \\
= & -[D F(f)]^{-1}\left[D^{2} F(f)\left([D F(f)]^{-1}(g),[D F(f)]^{-1}(h)\right)\right] .
\end{aligned}
$$

Define $H: B \rightarrow \mathcal{E}_{n}(C(X), C(X))$, space of all continuous symmetric $n$-linear maps of $C(X)^{n}$ into $C(X)$, by

$$
H(f)=[D F(f)]^{-1} D^{n} F(f)=D G(F(f)) D^{n} F(f) \text { for all } f \in B
$$


By the induction assumption $H(\alpha u)\left(u^{l}, k^{n-l}\right)\left(x_{0}\right)=0$ and $H(\alpha u+$ $\varepsilon u)\left(u^{l}, k^{n-l}\right)\left(x_{0}\right)=0$ for small $|\varepsilon|>0$. Hence

$$
\begin{aligned}
D H & (\alpha u)(u)\left(u^{l}, k^{n-l}\right)\left(x_{0}\right) \\
& =\lim _{\varepsilon \rightarrow 0} \frac{H(\alpha u+\varepsilon u)\left(u^{l}, k^{n-l}\right)\left(x_{0}\right)-H(\alpha u)\left(u^{l}, k^{n-l}\right)\left(x_{0}\right)}{\varepsilon}=0 .
\end{aligned}
$$

On the other hand,

$$
\begin{aligned}
D H(f)(u) & =D^{2} G(F(f))\left(D^{n} F(f), D F(f)(u)\right) \\
+ & D G(F(f))\left(D\left(D^{n} F(f)\right)(u)\right) \\
= & -[D F(f)]^{-1}\left[D^{2} F(f)(H(f), u)\right]+[D F(f)]^{-1}\left(D\left(D^{n} F(f)\right)(u)\right) .
\end{aligned}
$$

Since $H(\alpha u)\left(u^{l}, k^{n-l}\right) \in C(X)$ and vanishes at $x_{0}$, the first term is zero when evaluated at $f=\alpha u$ and $\left(u^{l}, k^{n-l}\right)$ and $x_{0}$ by Lemma 8.4. Hence

$$
\begin{aligned}
0 & =D H(\alpha u)(u)\left(u^{l}, k^{n-l}\right)\left(x_{0}\right) \\
& =[D F(\alpha u)]^{-1}\left(D\left(D^{n} F(\alpha u)\left(u^{l}, k^{n+1-(l+1)}\right)\right)(u)\right)\left(x_{0}\right)
\end{aligned}
$$

which is the result for $l+1$ and $n+1$.

LEMMA 8.6. Let $u \in C(X)$ such that $u\left(x_{0}\right)=1$ and $0<u(x)<1$ if $x \neq x_{0}$. If $f \in B$, then $F(f)\left(x_{0}\right)=F(u f)\left(x_{0}\right)$.

Proof. If $f\left(x_{0}\right)=0$, then $(u f)\left(x_{0}\right)=0$ and

$$
F(f)\left(x_{0}\right)=\sum_{n=1}^{\infty} \frac{1}{n !} D^{n} F(0)\left(f^{n}\right)\left(x_{0}\right)=f\left(x_{0}\right)
$$

since $f$ plays the role of $k$ in Lemma 8.3. Similarly, $F(u f)\left(x_{0}\right)=(u f)\left(x_{0}\right)$.

Assume $f\left(x_{0}\right) \neq 0$. Let $N=\left\{x \in X|u(x)<| f\left(x_{0}\right) \mid / 2\|f\|\right\}$ and set $v(x)=$ $u(x)$ if $x \in N$ and $v(x)=\min \left(u(x),\left|f\left(x_{0}\right)\right| /|f(x)|\right)$ if $x \in N^{c}$. Then $v \in$ $C(X)$ and $|(v f)(x)|<\frac{1}{2}\left|f\left(x_{0}\right)\right|$ if $x \in N$ and $|(v f)(x)|=\min \left(u(x)|f(x)|,\left|f\left(x_{0}\right)\right|\right)$ if $x \in N^{c}$. Therefore $v f / f\left(x_{0}\right)$ plays the role of $u$ in Lemma 8.5. Setting $\alpha=0$, in Lemma 8.5, we have, for all nonnegative integers $l<n$,

$$
D^{n} F(0)\left(\left(v f / f\left(v_{0}\right)\right)^{l},((1-v) f)^{n-l}\right)\left(x_{0}\right)=0
$$

and hence

$$
D^{n} F(0)\left((v f)^{l},((1-v) f)^{n-l}\right)\left(x_{0}\right)=0
$$


Then

$$
\begin{aligned}
F(f)\left(x_{0}\right) & =F(v f+(1-v) f)\left(x_{0}\right) \\
& =\sum_{n=1}^{\infty} \frac{1}{n !} D^{n} F(0)\left((v f+(1-v) f)^{n}\right)\left(x_{0}\right) \\
& =\sum_{n=1}^{\infty} \sum_{l=0}^{n} \frac{1}{l !(n-l) !} D^{n} F(0)\left((v f)^{l},((1-v) f)^{n-l}\right)\left(x_{0}\right) \\
& =\sum_{n=1}^{\infty} \frac{1}{n !} D^{n} F(0)\left((v f)^{n}\right)\left(x_{0}\right)=F(v f)\left(x_{0}\right) .
\end{aligned}
$$

If we use $u f$, instead of $f$, the same $v$ works for $u f$ and we have $F(u f)\left(x_{0}\right)=$ $F(v u f)\left(x_{0}\right)$. Since $v u$ can be used instead of $v$ for $f$, we have $F(f)\left(x_{0}\right)=$ $F(v u f)\left(x_{0}\right)$ and hence $F(f)\left(x_{0}\right)=F(u f)\left(x_{0}\right)$.

LEMMA 8.7. If $f, g \in B$ such that $f\left(x_{0}\right)=g\left(x_{0}\right)$, then $F(f)\left(x_{0}\right)=F(g)\left(x_{0}\right)$.

Proof. Let $\varepsilon>0$ be given. Since $F$ is continuous at $f$, there is $\delta>0$ such that $\|F(f)-F(h)\|<\varepsilon$ if $\|f-h\|<\delta$. Let $N_{1}=\{x \in X|| f(x)-g(x) \mid<$ $\delta$ \} and let $N$ be an open neighborhood of $x_{0}$ such that $\bar{N} \subset N_{1}$. Since $x_{0}$ is a $G_{\delta}$, there is $u \in C(X)$ such that $u\left(x_{0}\right)=1,0 \leqslant u(x)<1$, if $x \neq x_{0}$, and $u \equiv 0$ on $N_{1}^{c}$. By Urysohn's Lemma, there is $v \in C(X)$ such that $0 \leqslant v(x) \leqslant$ 1 for all $x \in X, v \equiv 1$ on $N$ and $v \equiv 0$ on $N_{1}^{c}$. Set $h=v g+(1-v) f$. Then $\|f-h\|<\delta$ and $u g=u h$. Therefore, by Lemma 8.6,

$$
\begin{aligned}
\left|F(f)\left(x_{0}\right)-F(g)\left(x_{0}\right)\right| & =\left|F(f)\left(x_{0}\right)-F(u g)\left(x_{0}\right)\right| \\
& =\left|F(f)\left(x_{0}\right)-F(u h)\left(x_{0}\right)\right| \\
& =\left|F(f)\left(x_{0}\right)-F(h)\left(x_{0}\right)\right| \leqslant\|F(f)-F(h)\|<\varepsilon .
\end{aligned}
$$

Since $\varepsilon$ can be made arbitrarily small, $F(f)\left(x_{0}\right)=F(g)\left(x_{0}\right)$.

We can now prove Theorem 8.1.

Proof. Let $f, g \in B$ and $x_{0} \in X$. For small $|\alpha|$, we have that $f+\alpha g$, $f+\alpha g\left(x_{0}\right) 1 \in C(X)$ and agree at $x_{0}$; therefore, by Lemma 8.7, $F(f+$ $\alpha g)\left(x_{0}\right)=F\left(f+\alpha g\left(x_{0}\right) 1\right)$. Therefore,

$$
\begin{aligned}
D F(f)(g)\left(x_{0}\right) & =\lim _{\alpha \rightarrow 0} \frac{F(f+\alpha g)\left(x_{0}\right)-F(f)\left(x_{0}\right)}{\alpha} \\
& =\lim _{\alpha \rightarrow 0} \frac{F\left(f+\alpha g\left(x_{0}\right) 1\right)\left(x_{0}\right)-F(f)\left(x_{0}\right)}{\alpha} \\
& =D F(f)\left(g\left(x_{0}\right) 1\right)\left(x_{0}\right)=g\left(x_{0}\right) D F(f)(1)\left(x_{0}\right) .
\end{aligned}
$$

Since $x_{0}$ is arbitrary, we have $D F(f)(g)=g D F(f)(1)$; i.e., $F$ is $L$-analytic in $B$ and $F^{\prime}(f)=D F(f)(1)$. 
RemarK 1. The normalization $D F(0)=I$ is necessary in Theorem 8.1 as is seen in the following example. Define $F: C[0,1] \rightarrow C[0,1]$ by $F(f)(x)=\left(x+\frac{1}{2}\right) f(x)+\left(\frac{1}{2}-x\right) f\left(x+\frac{1}{2}\right)$ if $x \in\left[0, \frac{1}{2}\right)$ and $F(f)(x)=f(x)$ if $x \in\left[\frac{1}{2}, 1\right]$. Then $F$ is a continuous linear map of $C[0,1]$ onto $C[0,1]$ and so $F$ is a convex biholomorphic function in $B$. But $F$ is not $L$-analytic and $D F(0)=F \neq I$.

Without the normalization, we have

COROLlaRY. If $F$ is biholomorphic in $B$, and $F(B)$ is convex then $F=L \circ G$ where $L$ is a univalent affine map of $C(X)$ onto $C(X)$ and $G$ is bianalytic in $B$.

Proof. Define $L(f)=F(0)+D F(0)(f)$ for all $f \in B$ and $G=L^{-1} \circ F$ satisfies Theorem 8.1.

REMARK 2. The proof of Theorem 8.1 depends on the existence of a peaking function $u$ at each point $x \in X$. In general, an arbitrary compact $T_{2}$-space $X$ does not have peaking functions at each point; for example, if $X$ is the set of all ordinals which are less than or equal to the first uncountable ordinal with the order topology, then the first uncountable ordinal is not a $G_{\delta}$ point. See [10, Exercises 1.I, 5.C, and 4.J]. It would be interesting to know if Theorem 8.1 is true for an arbitrary compact $T_{2}$-space.

Note that Theorem 8.1 contains Theorem 3 of [15]. To see this, take $X=\{1,2, \ldots, n\}$ with the discrete topology. Also compare Theorem 8 of [16].

9. Example and a remark. We now give an example of a function which is univalent and $F$-holomorphic in $B$ such that $F^{-1}$ is not $F$-holomorphic in $F(B), F(B)$ contains an open set, but $F(B)$ is not open. The example is in the Banach algebra $H^{\infty}=\{f \mid f: \Delta \rightarrow \mathbf{C}$ is holomorphic in $\Delta$ and $\sup \{|f(z)| \mid z \in$ $\Delta\}<\infty$ [ [3], [8]. Define $F: B \rightarrow H^{\infty}$ by $F(f)=f+a f^{2}$ for nonconstant $a \in H^{\infty}$ such that for some $z_{0} \in \Delta,\left|a\left(z_{0}\right)\right|=\frac{1}{2}$. To show that $F$ is univalent in $B$, suppose that $f, g \in B$ such that $F(f)=F(g)$. Then $(f-g)(1+a(f+$ $g))=0$ which implies that $f(z)=g(z)$ or $f(z)+g(z)=-1 / a(z)$ for each $z \in \Delta$. We claim the first equation always holds. By hypothesis, there is $z_{0} \in \Delta$ such that $\left|a\left(z_{0}\right)\right|=\frac{1}{2}$. Since $f, g \in B$, there is $\delta>0$ such that $|f(z)|<$ $1-\delta$ and $|g(z)|<1-\delta$ in some open neighborhood of $z_{0}$. The second equation implies $1 /|a(z)|<2-2 \delta$ in this neighborhood, hence $\left|a\left(z_{0}\right)\right|>1 /(2-2 \delta)>\frac{1}{2}$, which is a contradiction. Hence $f(z)=g(z)$ in this open neighborhood of $z_{0}$ and therefore $f=g$.

To prove that $F^{-1}$ is not $F$-holomorphic in $F(B)$, observe that $F^{\prime}(f)=1+$ 2 af and so $F^{\prime}(f)$ is nonsingular as long as $1+2 a(z) f(z) \neq 0$ for $z \in \Delta$. If $\left|a\left(z_{1}\right)\right|>\frac{1}{2}$, let $f$ be the constant function $-1 / 2 a\left(z_{1}\right)$. Then $\|f\|<1$ and $F^{\prime}(f)$ is singular. It follows that $F^{-1}$ is not $F$-holomorphic in $F(B)$.

$F(B)$ contains an open set since the equation $\varepsilon=f+a f^{2}$ has the solution 
$f=(-1+\sqrt{1+4 a \varepsilon}) / 2 a=2 \varepsilon /(1+\sqrt{1+4 a \varepsilon})$ where $\varepsilon \in H^{\infty}$ and $\|\varepsilon\|$ is small.

To prove that $F(B)$ is not open, suppose $\left|a\left(z_{1}\right)\right|>\frac{1}{2}$ and let $f$ be the constant function $-1 / 2 a\left(z_{1}\right)$. Then $F(f)=a / 4 a^{2}\left(z_{1}\right)-1 / 2 a\left(z_{1}\right)$, and if $\varepsilon \in H^{\infty},\|\varepsilon\|$ small, we have $a / 4 a^{2}\left(z_{1}\right)-1 / 2 a\left(z_{1}\right)+\varepsilon=f+a f^{2}$ for some $f \in B$ if and only if $f=-1 / 2 a\left(z_{1}\right)+\delta$ where $\delta \in H^{\infty}$ and $\varepsilon(z)=\delta(z)(1$ $\left.-a(z) / a\left(z_{1}\right)\right)+a(z) \delta^{2}(z)$. Let $\varepsilon$ have the property that $\varepsilon\left(z_{1}\right)=0$. Then $0=\varepsilon\left(z_{1}\right)=a\left(z_{1}\right) \delta^{2}\left(z_{1}\right)$ so we conclude $\delta\left(z_{1}\right)=0$. Since $\varepsilon(z)=\delta(z)(1-$ $\left.a(z) / a\left(z_{1}\right)\right)+a(z) \delta^{2}(z)$, it follows that $\varepsilon$ has at least a double zero at $z_{1}$. This means that any function of the form $a / 4 a^{2}\left(z_{1}\right)-1 / 2 a\left(z_{1}\right)+\varepsilon$ such that $\varepsilon\left(z_{1}\right)=0$ and $\varepsilon^{\prime}\left(z_{1}\right) \neq 0$ cannot lie in $F(B)$.

This example shows that Theorem $5[5]$ is false. $F$ is univalent and $F$-holomorphic in $B$ with $F(0)=0$ and $D F(0)=I$, but $F(B)$ is not open. Hence $F$ is not locally biholomorphic in $B$ and therefore $F$ is not $\Phi$-like for any function $\Phi$.

\section{REFERENCES}

1. Louis Brickman, $\Phi$-like analytic functions. I, Bull. Amer. Math. Soc. 79 (1973), 555-558.

2. James Dugundji, Topology, Allyn and Bacon, Boston, Mass., 1966.

3. Peter L. Duren, Theory of $\boldsymbol{H}^{p}$ spaces, Academic Press, New York, 1970.

4. I. Gelfand, D. Raikov and G. Shilov, Commutative normed rings, Chelsea, New York, 1964.

5. Kenneth R. Gurganus, $\Phi$-like holomorphic functions in $\mathbf{C}^{\mathbf{n}}$ and Banach spaces, Trans. Amer. Math. Soc. 205 (1975), 389-406.

6. Einar Hille, Methods in classical and functional analysis, Addison-Wesley, Reading, Mass., 1972.

7. Einar Hille and Ralph S. Phillips, Functional analysis and semi-groups, Amer. Math. Soc. Colloq. Publ., vol. 31, rev. ed., Amer. Math. Soc., Providence, R. I., 1957.

8. Kenneth Hoffman, Banach spaces of analytic functions, Prentice-Hall, Englewood Cliffs, N. J., 1962.

9. Wilfred Kaplan, Close-to-convex schlicht functions, Michigan Math. J. 1 (1952), 169-185.

10. John L. Kelley, General topology, Van Nostrand, Princeton, N. J., 1955.

11. Edgar $\mathrm{R}$. Lorch, The theory of analytic functions in normed abelian vector rings, Trans. Amer. Math. Soc. 54 (1943), 414-425.

12. J. A. Pfaltzgraff, Subordinate chains and univalence of holomorphic mappings on $\mathbf{C}^{n}$, Math. Ann. 210 (1974), 55-68.

13. J. A. Pfaltzgraff and T. J. Suffridge, Close-to-starlike holomorphic functions of several variables, Pacific J. Math. 57 (1975), 271-279.

14. L. Spaček, Contribution à la théorie des fonctions univalentes, Časopis Pèst. Mat.-Fys. 62 (1933), $12-19$.

15. T. J. Suffridge, The principle of subordination applied to functions of several variables, Pacific J. Math. 33 (1970), 241-248.

16. __ Starlike and convex maps in Banach spaces, Pacific J. Math. 46 (1973), 575-589.

17. , Starlikeness, convexity and other geometric properties of holomorphic maps in higher dimensions, Lecture Notes in Math., vol. 599, Springer-Verlag, Berlin and New York, 1977, pp. 146-159.

Department of Mathematics, University of Kentucky, Lexington, Kentucky 40506 (Current address of T. J. Suffridge)

Current address (L. F. Heath): Department of Mathematics, University of Texas at Arlington, Arlington, Texas 76019 